\title{
Achyranthes bidentata polysaccharides activate the Wnt/ $\beta$-catenin signaling pathway to promote chondrocyte proliferation
}

\author{
XIAPING WENG ${ }^{1 *}$, PINGDONG LIN ${ }^{1 *}$, FAYUAN LIU ${ }^{2}$, JIASHOU CHEN $^{1}$, HUITING LI $^{1}$, \\ LICHAN HUANG $^{3}$, CHUNSONG ZHEN $^{2}$, HUIFENG XU' ${ }^{2}$, XIANXIANG LIU ${ }^{2}$, HONGZHI YE $^{2}$ and XIHAI LI ${ }^{2}$ \\ ${ }^{1}$ College of Pharmacy, ${ }^{2}$ Academy of Integrative Medicine, ${ }^{3}$ Fujian Key Laboratory of Integrative Medicine on Geriatrics, \\ Fujian University of Traditional Chinese Medicine, Fuzhou, Fujian 350122, P.R. China
}

Received May 17, 2014; Accepted July 15, 2014

DOI: $10.3892 /$ ijmm.2014.1869

\begin{abstract}
Achyranthes bidentata polysaccharides (ABPS) are the active components of Radix Achyranthis Bidentatae (AB), which has been extensively used in Traditional Chinese medicine (TCM) in the treatment of osteoarthritis (OA). Our previous study provided evidence that ABPS regulated the $\mathrm{G} 1 / \mathrm{S}$ transition to promote chondrocyte proliferation. However, the precise mechanisms involved remain to be elucidated. In the present study, we aimed to investigate the effects of ABPS on the Wnt//-catenin signaling pathway in chondrocytes. Chondrocytes, obtained from the knee cartilage of Sprague-Dawley rats, were identified by type II collagen immunohistochemistry. ABPS upregulated the expression of Wnt-4, Frizzled-2, $\beta$-catenin and cyclin D1, and downregulated the expression of glycogen synthase kinase $3 \beta$ (GSK-3 $\beta$ ), as shown by reverse transcription PCR (RT-PCR) and western blot analysis. Using immunofluorescence, we also found that ABPS induced $\beta$-catenin nuclear translocation. Importantly, the expression of $\beta$-catenin and cyclin D1 was partly inhibited by Dickkopf-1 (DKK-1), an inhibitor of the Wnt/3-catenin signaling pathway. In addition, we found that ABPS increased the expression of type II collagen in chondrocytes. These results suggest that ABPS promote chondrocyte proliferation by activating the $\mathrm{Wnt} / \beta$-catenin signaling pathway.
\end{abstract}

Correspondence to: Professor Hongzhi Ye or Dr Xihai Li, Academy of Integrative Medicine, Fujian University of Traditional Chinese Medicine, 1 Huatuo, University Town, Shangjie Minhou, Fuzhou, Fujian 350122, P.R. China

E-mail: yelin0930@163.com

E-mail: lixihai79dahai@163.com

${ }^{*}$ Contributed equally

Key words: Achyranthes bidentata polysaccharides, Wnt/ $\beta$-catenin signaling pathway, chondrocyte, osteoarthritis

\section{Introduction}

Osteoarthritis (OA), one of the most chronic diseases affecting the joint cartilage of middle-aged and elderly individuals, is characterized by the degradation of articular cartilage (1). Chondrocytes, the only type of cell found in cartilage, controls the balance of catabolism and anabolism to maintain the structural and functional integrity of the extracellular matrix (ECM) (2-4). As cartilage has a poor repair and regeneration capacity, enhancing chondrocyte proliferation may contribute to the inhibition of the development and progression of $\mathrm{OA}(5)$.

The Wnt/ $\beta$-catenin signaling pathway plays a crucial role in the processes of cell proliferation (6). Following the activation of the $\mathrm{Wnt} / \beta$-catenin signaling pathway, $\beta$-catenin accumulates in the cytosol, and then translocates to the nucleus, subsequently binding to the transcription factors, T-cell factor (TCF) and lymphoid enhancer factor (LEF) (7). These factors accelerate cell cycle progression by regulating the expression of cyclin D1, an important factor in the cell cycle.

Radix Achyranthis Bidentatae (AB), has been widely used in traditional Chinese medicinal formulations for the clinical treatment of OA $(8,9)$. In a previous study of ours, we demonstrated that Achyranthes bidentata polysaccharides (ABPS) extracted from $\mathrm{AB}$ induce chondrocyte proliferation by promoting the G1/S transition (10). However, the specific mechanisms involved remain to be fully elucidated. In the present study, we aimed to determine whether ABPS promote chondrocyte proliferation by activating the Wnt/ $\beta$-catenin signaling pathway.

\section{Materials and methods}

Animals. Male Sprague-Dawley (SD) rats at 6 weeks of age were purchased from Shanghai SLAC Laboratory Animal Co., Ltd. (Shanghai, China). The care and use of the animals in the present study were strictly according to the Guidance Suggestions for the Care and Use of Laboratory Animals administered by the Ministry of Science and Technology of China and the Fujian University of Traditional Chinese Medicine, Fuzhou, China.

Preparation of ABPS. The method used to extract ABPS was the one used in our previous study (10). The extracted 


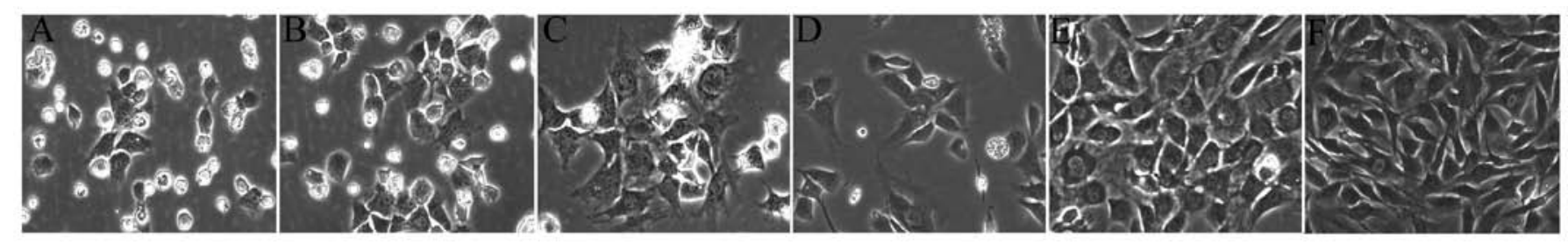

Figure 1. Morphology and identification of chondrocytes (original magnification, x200). Primary chondrocytes cultured for (A) 2 days, (B) 3 days and (C) 5 days. The first passage chondrocytes cultured for (D) 1 day and (E) 3 days. The second passage chondrocytes cultured for (F) 3 days.

ABPS were dissolved in Dulbecco's modified Eagle's medium (DMEM; HyClone, Logan, UT, USA) containing 10\% fetal bovine serum (FBS; HyClone), then filtered through a $0.22-\mu \mathrm{m}$ filter and stored at $4^{\circ} \mathrm{C}$.

Isolation, culture and identification of chondrocytes. Rat articular chondrocytes were isolated and cultured as previously described (11). Passage (P) 2 chondrocytes were identified by type II collagen immunohistochemistry. The chondrocytes were first treated with 50, 100 and $200 \mu \mathrm{g} / \mathrm{ml} \mathrm{ABPS}$ for $48 \mathrm{~h}$, as previously described (10). In order to further verify the mechanisms involved, the chondrocytes were treated with $0.2 \mu \mathrm{g} / \mathrm{ml}$ Dickkopf-1 (DKK-1; R\&D Systems, Minneapolis, MN, USA) and treated with ABPS $(100 \mu \mathrm{g} / \mathrm{ml})$ in the presence or absence of DKK-1 for $48 \mathrm{~h}$, as previously described $(10,12)$.

Western blot analysis. Following treatment, the proteins were collected immediately in lysis buffer and quantified using the BCA method. Proteins $(20 \mu \mathrm{g})$ were separated on a $12 \%$ SDS-PAGE gel and transferred onto PVDF membranes. After transferring, the membranes were blocked and incubated overnight at $4^{\circ} \mathrm{C}$ with the following primary antibodies $(1: 1,000)$ : Wnt-4, Frizzled-2 (Santa Cruz Biotechnology, Inc., Santa Cruz, CA, USA), $\beta$-catenin, glycogen synthase kinase $3 \beta(\mathrm{GSK}-3 \beta)$ (Cell Signaling Technology, Inc., Beverly, MA, USA), cyclin D1, type II collagen (Bio-Word Technology, Natong, China) or $\beta$-actin (Santa Cruz Biotechnology). Horseradish peroxidase (HRP)-conjugated secondary antibody (Beijing Zhongshan Golden Bridge Biotechnology Co., Ltd., Beijing, China) was then added to the membranes at room temperature. The immunocomplexes were visualized using the ECL method. $\beta$-actin was used as an internal control.

RNA extraction and RT-PCR analysis. Total RNA was extracted using TRIzol reagent (Invitrogen, Grand Island, NY, USA), and then quantified followed by being reverse transcribed into cDNA. We performed PCR to determine the mRNA levels of Wnt-4, $\beta$-catenin, Frizzled-2, GSK-3 $\beta$, cyclin D1 and type II collagen. The primers used for PCR were as follows: Wnt-4 forward, 5'-TCA GCC CAC AGG GTT TCC A-3' and reverse, 5'-CGC TCG CCA GCA TGT CTT T-3'; $\beta$-catenin forward, 5'-AAG GAA GCT TCC AGA CAT GC-3' and reverse, 5'-AGC TTG CTC TCT TGA TTG CC-3'; Frizzled-2 forward, 5'-TCG AGG CCA ATT CGC AGT A-3' and reverse, 5'-CAG GAA GGA TGT GCC GAT G-3'; GSK-3 $\beta$ forward, 5'-AAA GTG CAT CGC TGG CTT A-3' and reverse, 5'-GTC GAC GGT TTG TTT CCA AT-3'; cyclin D1 forward, 5'-AAT GCC AGA GGC GGA TGA GA-3' and reverse, 5'-GCT TGT GCG GTA GCA GGA GA-3'; type II collagen forward, 5'-CCA GAG TGG
AAG AGC GGA GAC-3' and reverse, 5'-CAG TGG ACA GTA GAC GGA GGA AAG-3'; and $\beta$-actin forward, 5'-CAC CCG CGA GTA CAA CCT TC-3' and reverse, 5'-CCC ATA CCC ACC ATC ACA CC-3'. The DNA bands were examined using a Gel Documentation system (Model Gel Doc 2000; Bio-Rad Laboratories, Hercules, CA, USA). $\beta$-actin was used as an internal control.

Immunofluorescence staining. Following treatment with ABPS, the cells were fixed in ice-cold methanol and permeabilized with $1 \%$ Triton X-100 for $10 \mathrm{~min}$. The cells were blocked with $5 \%$ bovine serum albumin, then incubated with rabbit anti- $\beta$-catenin antibody overnight at $4{ }^{\circ} \mathrm{C}$ followed by incubation with TRITC-conjugated secondary antibody (Zymed Laboratories, San Francisco, CA, USA) and DAPI staining. The signal was visualized and images were acquired using a fluorescence microscope (Olympus, Tokyo, Japan).

Statistical analysis. Data are expressed as the means \pm standard deviation. The data were processed using SPSS software version 18.0 with the Student's-test or ANOVA. A value of $\mathrm{P}<0.05$ was considered to indicate a statistically significant difference.

\section{Results}

Morphology and identification of chondrocytes. The morphology of the chondrocytes has been described in previous studies $(10,11)$; the cultured chondrocytes in this study had the typical characteristics of chondrocytes (Fig. 1). The primary chondrocytes were small, round cells when first suspended in DMEM. After being cultured for 2 days, the volumes of cells nestled against the culture flask became larger and a small number of cells began to elongate and form protuberances (Fig. 1A). Three days later, the cells showed a fusiform or oval shape with a clear outline (Fig. 1B). Subsequent to 5 days of proliferation, the cells grew to be tufted (Fig. 1C). The P1 and P2 chondrocytes spread across the flask much more rapidly (Fig. 1D, E and F). The morphology of the P2 chondrocytes showed more typical characteristics of chondrocytes and the chondrocytes contained abundant levels of type II collagen, a major secretory molecule of the cartilage extracellular matrix (ECM). Compared with the negative control, which was not treated with type II collagen antibody, the cytoplasm was stained brown, which represented the positive expression of type II collagen in chondrocytes (Fig. 4G and H).

ABPS increase the expression of Wnt-4, Frizzled-2, $\beta$-catenin

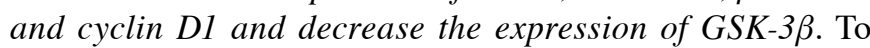
determine the effects of ABPS on the Wnt/ $\beta$-catenin signaling 

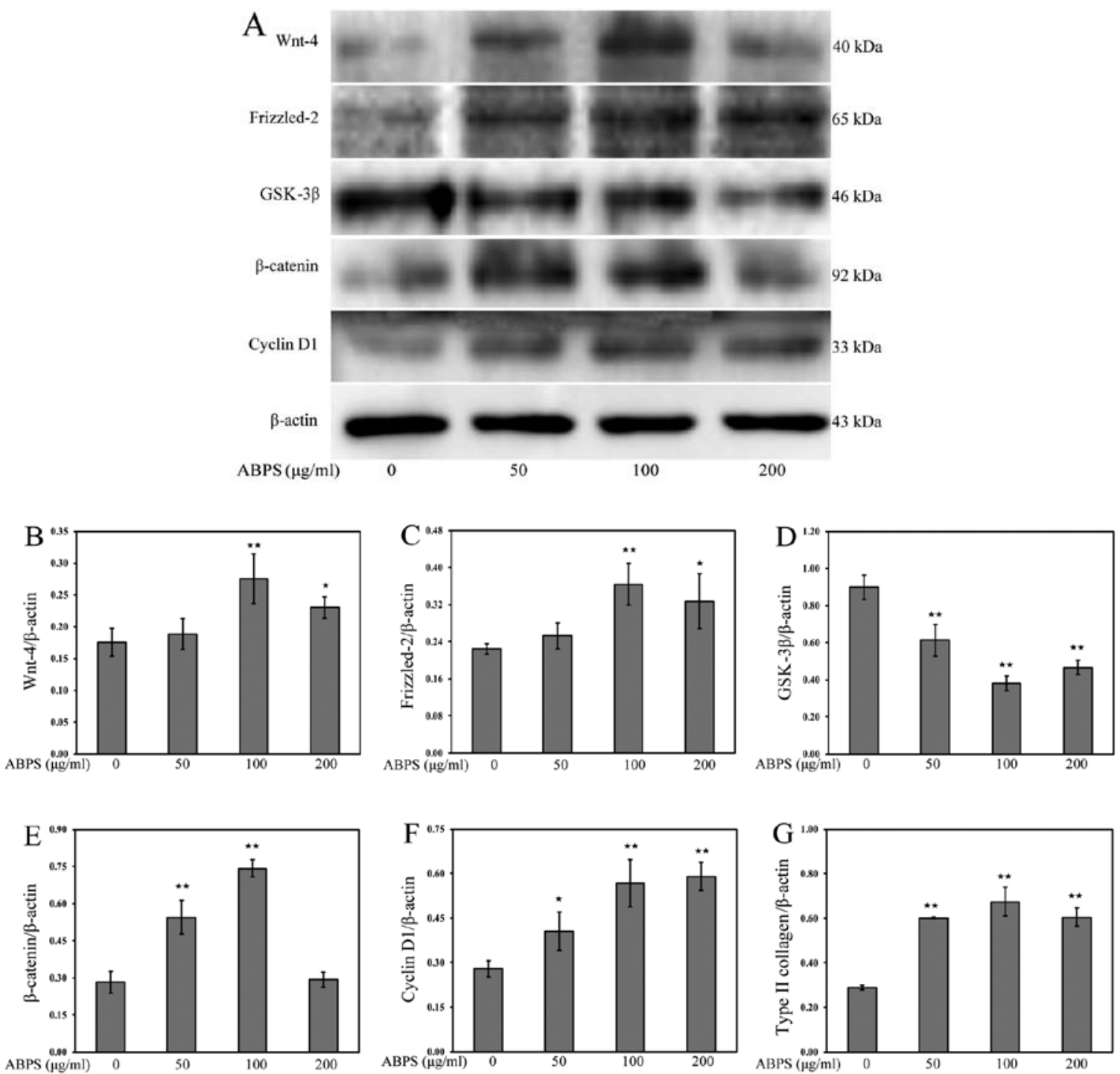

Figure 2. Protein levels of Wnt-4, Frizzled-2, glycogen synthase kinase (GSK)-3 $\beta, \beta$-catenin, cyclin D1 and type II collagen in chondrocytes following treatment with Achyranthes bidentata polysaccharides (ABPS). (A) Western blots showing the protein levels of Wnt-4, Frizzled-2, GSK-3 $\beta$, $\beta$-catenin, cyclin D1 and collagen II. $\beta$-actin was used as the internal control. (B-G) Quantification of the protein levels of Wnt-4, Frizzled-2, GSK-3 $\beta$, $\beta$-catenin, cyclin D1 and type II collagen by western blot analysis. ${ }^{* *} \mathrm{P}<0.01,{ }^{*} \mathrm{P}<0.05$, compared with the control group (untreated cells).

pathway in chondrocytes, we used RT-PCR and western blot analysis to examine the mRNA and protein expression of Wnt-4, Frizzled-2, $\beta$-catenin, GSK-3 $\beta$ and cyclin D1. Compared with the control group (untreated cells), the protein levels of Wnt-4, Frizzled-2, $\beta$-catenin and cyclin D1 in the ABPS-treated chondrocytes were significantly upregulated $(\mathrm{P}<0.01$ and $\mathrm{P}<0.05)$, while the protein level of GSK-3 $\beta$ was significantly downregulated $(\mathrm{P}<0.01$ and $\mathrm{P}<0.05)$ (Fig. $2 \mathrm{~A}-\mathrm{F})$. The mRNA expression of Wnt-4, Frizzled-2, $\beta$-catenin, GSK-3 $\beta$ and cyclin D1 was similar to their respective protein levels (Fig. 3A-F).

ABPS promote $\beta$-catenin nuclear translocation. The effects of ABPS on the nuclear translocation of $\beta$-catenin were further confirmed by immunouorescence staining. Immunouorescence staining revealed that ABPS markedly promoted the translocation $\beta$-catenin into the nucleus (Fig. $4 \mathrm{~A}-\mathrm{F}$ ). $\beta$-catenin mainly existed in the cytoplasm in the untreated cells; however, following treatment with ABPS, the staining was more intense and localized in the nucleus. These results demonstrated that
ABPS activated the Wnt/ $\beta$-catenin signaling pathway by promoting the nuclear localization of $\beta$-catenin.

The expression of cyclin D1 and $\beta$-catenin is partly decreased following the inhibition of the Wnt/ $\beta$-catenin singaling pathway. To further confirm that ABPS promote chondrocyte proliferation through the $\mathrm{Wnt} / \beta$-catenin singaling pathway, DKK-1, an inhibitor of the Wnt/ $\beta$-catenin receptor, was added to block the activation of the $\mathrm{Wnt} / \beta$-catenin signaling pathway. The results revealed that the expression of cyclin D1 and $\beta$-catenin was partly inhibited $(\mathrm{P}<0.01$ and $\mathrm{P}<0.05)$, indicating that ABPS participate in the regulation of chondrocyte proliferation through the $\mathrm{Wnt} / \beta$-catenin singaling pathway, and perhaps also through other channels (Fig. 5).

ABPS increase the expression of type II collagen. The development of articular cartilage degradation results in the loss of type II collagen, a main constituent of articular cartilage $(13,14)$; thus, in the present study, we examined the levels of type II 

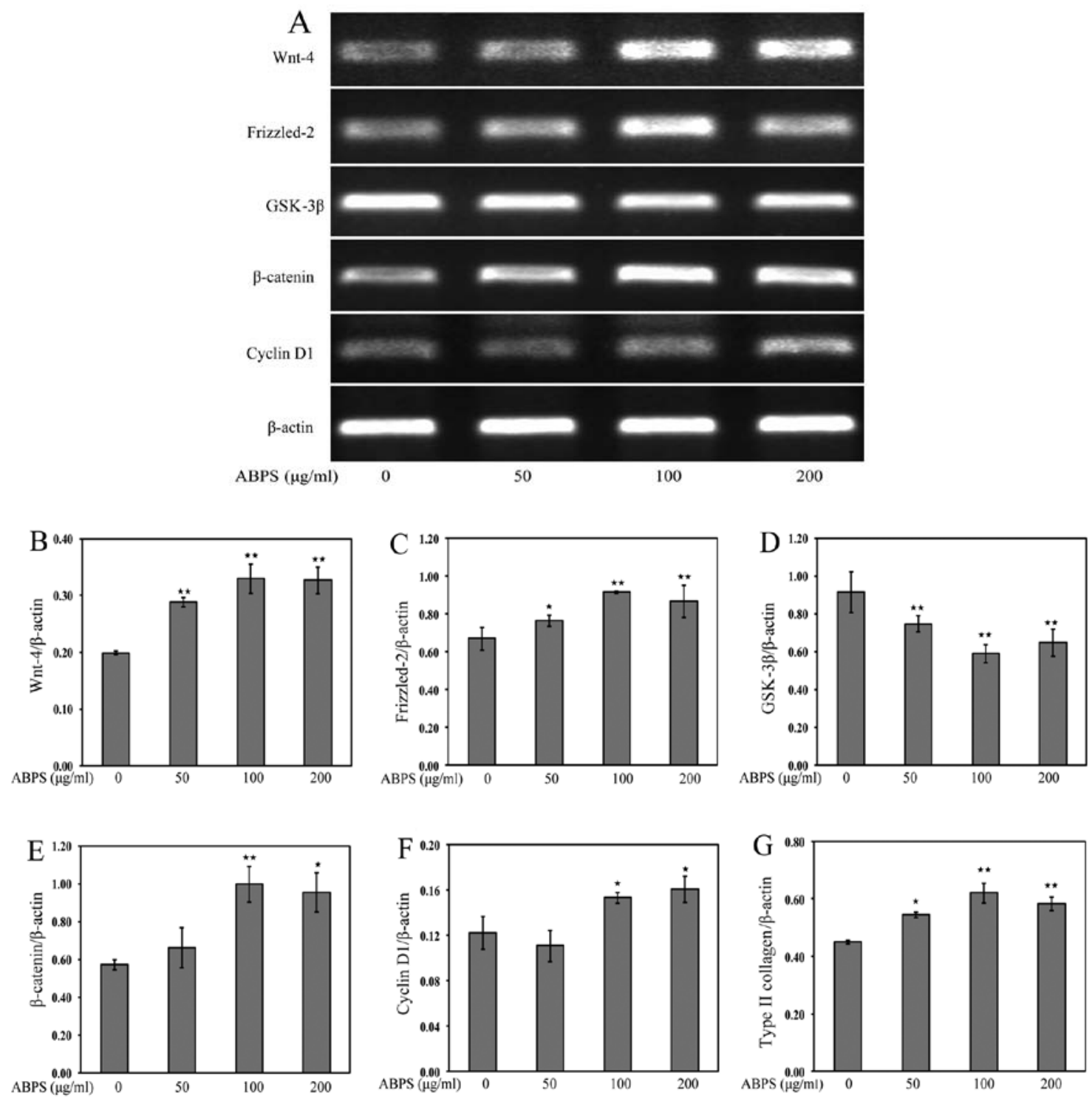

Figure 3. Achyranthes bidentata polysaccharides (ABPS) increase the mRNA expression of Wnt-4, Frizzled-2, $\beta$-catenin, cyclin D1 and type II collagen, and decrease the expression of glycogen synthase kinase (GSK-3 $\beta$ ). (A) mRNA expression of Wnt-4, Frizzled-2, GSK-3 $\beta$, $\beta$-catenin, cyclin D1 and collagen II analyzed by RT-PCR. (B-G) Quantification of mRNA expression of Wnt-4, Frizzled-2, GSK-3 $\beta, \beta$-catenin, cyclin D1 and type II collagen. ${ }^{* *} \mathrm{P}<0.01,{ }^{*} \mathrm{P}<0.05$, compared with the control group (untreated cells).

collagen in the chondrocytes. The results revealed that the expression of type II collagen was significantly increased compared with the control group (untreated cells) $(\mathrm{P}<0.01$ and $\mathrm{P}<0.05$ ) (Figs. 6, 2G and 3G), indicating that ABPS promoted chondrocyte proliferation by increasing the expression of type II collagen, a major secretory molecule of the ECM.

\section{Discussion}

Polysaccharides, a type of macromolecule with a broad range of biological activities, have attracted attention as they play a crucial role in human diseases $(15,16)$. Recently, studies have demonstrated that polysaccharides contribute to chondrocyte proliferation $(10,11)$. Thus, polysaccharides may be a potentially novel therapeutic target for OA by promoting chondrocyte proliferation. Our results revealed that ABPS upregulated the expression of Wnt-4, Frizzled-2, $\beta$-catenin and cyclin D1, and downregulated the expression of GSK-3 $\beta$.
ABPS induced the translocation of $\beta$-catenin into the nucleus in the chondrocytes. Furthermore, the expression of $\beta$-catenin and cyclin D1 was partly decreased following the addition of DKK-1, indicating that ABPS activated the Wnt/ $\beta$-catenin signaling pathway to promote chondrocyte proliferation.

Currently, western pharmacological intervention that addresses chronic pain in $\mathrm{OA}$ is frequently insufficient or poorly tolerated $(17,18)$. Non-steroidal anti-inflammatory drugs (NSAIDs) are the most common treatments for OA $(19,20)$. However, NSAIDs have a wide variety of side-effects. There are growing interests in herbal medicines, which seem to have an encouraging risk-benefit profile (21). ABPS, extracted from $\mathrm{AB}$, are one of the most effective natural elements used for the treatment of OA.

The Wnt/ $\beta$-catenin signaling pathway is important to the regulation of proliferation (22). The Wnt//-catenin pathway is activated when Wnt proteins bind to Frizzled family receptors and low density lipoprotein receptor-related protein (LRP) 5/6, which 

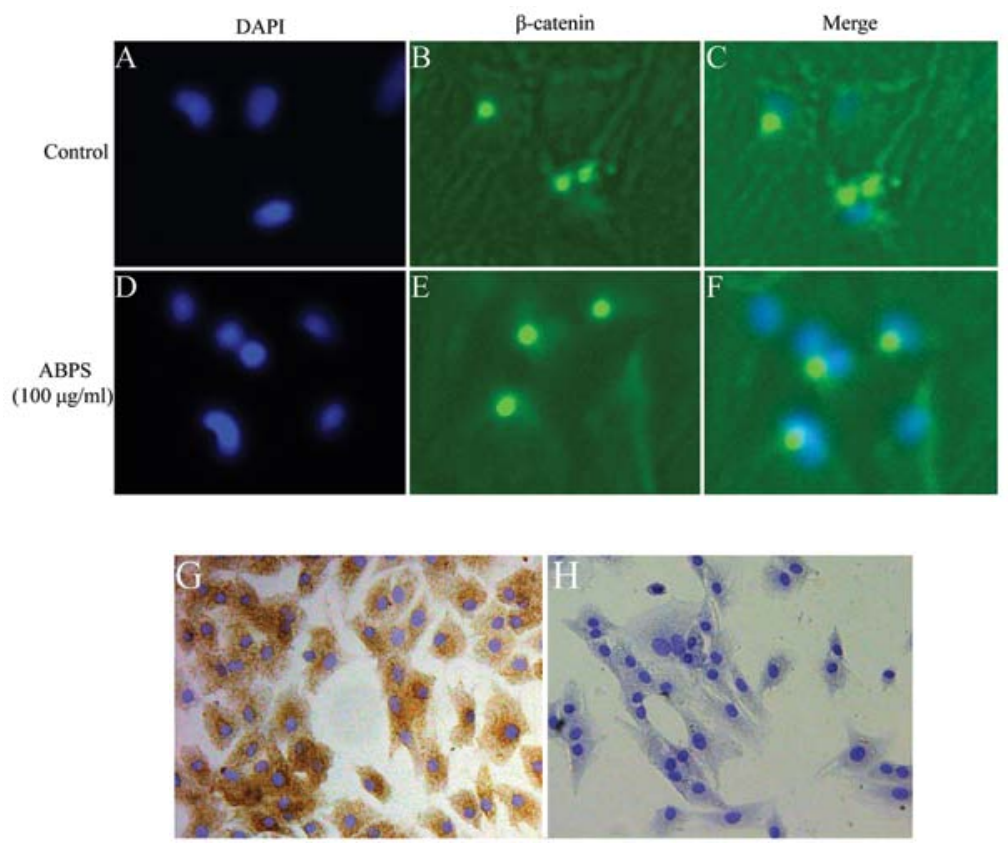

Figure 4. Achyranthes bidentata polysaccharides (ABPS) promote $\beta$-catenin nuclear translocation. Chondrocytes were treated with $100 \mu \mathrm{g} / \mathrm{ml} \mathrm{ABPS} \mathrm{for} 48 \mathrm{~h}$, followed by immunofluorescence staining for $\beta$-catenin (green). The nuclei were stained with DAPI (blue). (A-F) $\beta$-catenin immunostaining was predominantly localized in the nucleus following treatment with ABPS. (G) The second passage chondrocytes cultured for 4 days were identified by type II collagen immunohistochemistry. (H) The negative control was identified by type II collagen immunohistochemistry.
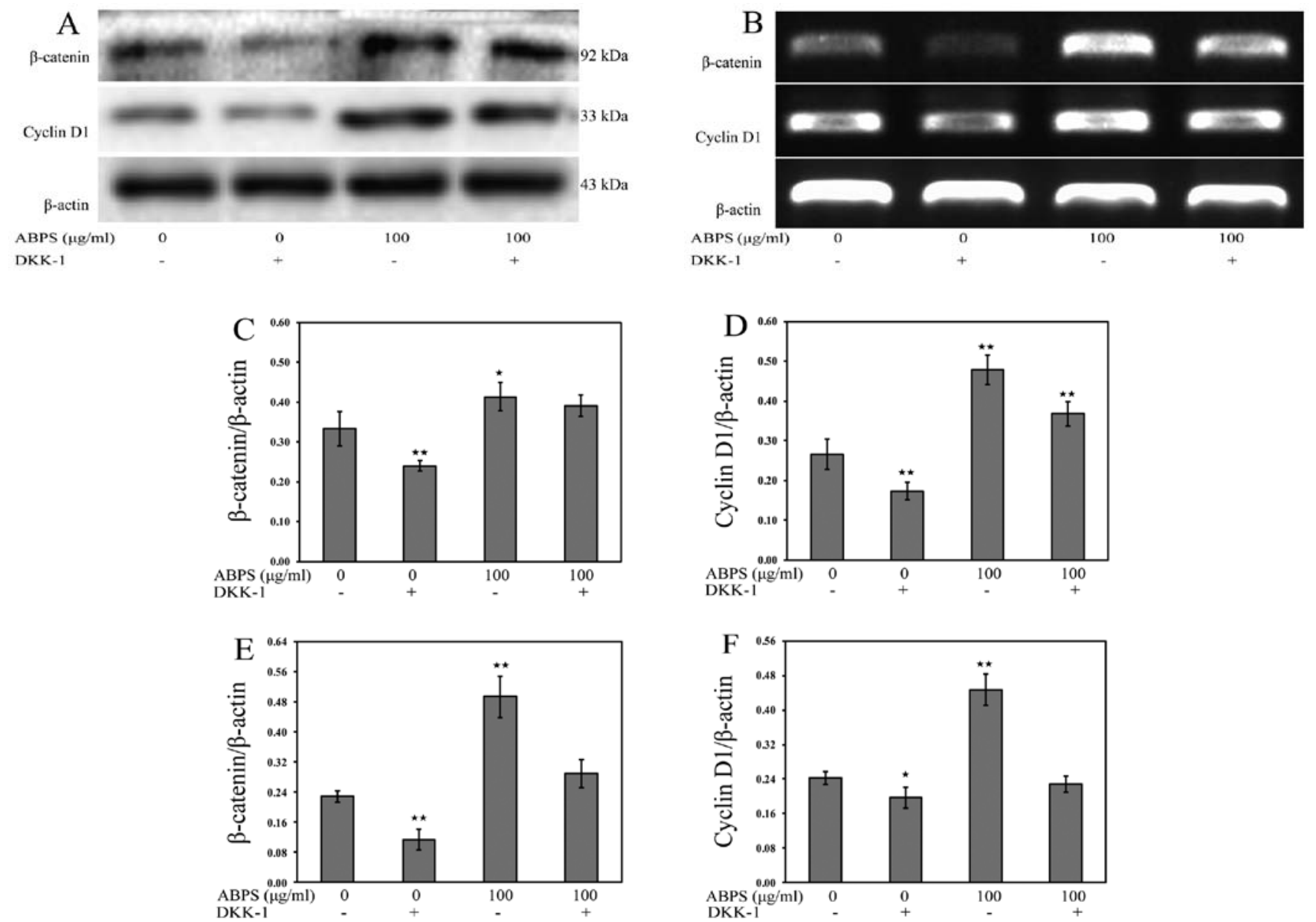

Figure 5. Inhibitory effect of Dickkopf-1 (DKK-1) on the Achyranthes bidentata polysaccharides (ABPS)-induced expression of cyclin D1 and $\beta$-catenin. Chondrocytes were treated with $0.2 \mu \mathrm{g} / \mathrm{ml}$ DKK-1 and incubated with ABPS $(100 \mu \mathrm{g} / \mathrm{ml})$ in the presence or absence of DKK-1. (A) Protein levels of $\beta$-catenin and cyclin D1. (B) mRNA expression of $\beta$-catenin and cyclin D1. (C-F) Protein and mRNA expression of $\beta$-catenin and cyclin D1. ${ }^{* *} \mathrm{P}<0.01,{ }^{*} \mathrm{P}<0.05$, compared with the control group (untreated cells). 


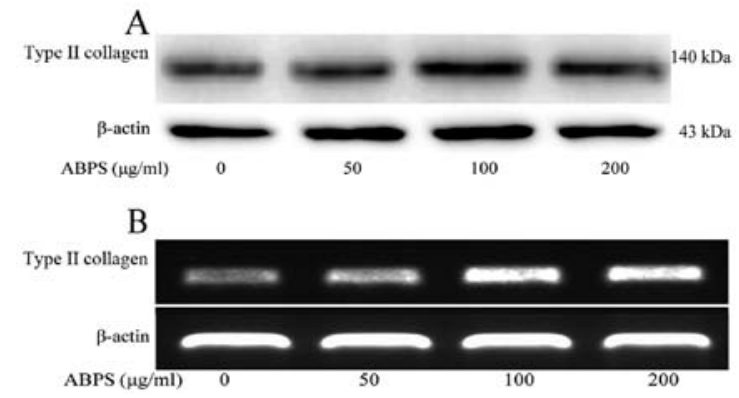

Figure 6. Achyranthes bidentata polysaccharides (ABPS) increase the expression of type II collagen in chondrocytes. (A and B) Protein and mRNA expression of type II collagen.

results in the activation of dishevelled (Dvl) family proteins (23). The activation of Dvl leads to the inhibition of GSK-3 $\beta$ that causes non-phosphorylated $\beta$-catenin to accumulate in the cytoplasm and migrate to the nucleus (24). Subsequently, $\beta$-catenin interacts with transcription factors, thus altering the expression of $\mathrm{Wnt} / \beta$ catenin signaling target genes, such as cyclin D1, that is a positive regulator of the G1/S transition. Our results demonstrated that ABPS increased the expression of Wnt-4, Frizzled-2, $\beta$-catenin and cyclin D1, whereas it decreased the expression of GSK-3 $\beta$, and significantly promoted the translocation of $\beta$-catenin into the nucleus. These results suggest that ABPS activate the Wnt/ $\beta$ catenin signaling pathway by inhibiting GSK-3 $\beta$ to promote the translocation of non-phosphorylated $\beta$-catenin into the nucleus to enhance cyclin D1 expression.

DKK-1 binds to LRP5/6 on target cells, resulting in the inhibition of $\mathrm{Wnt} / \beta$-catenin signaling pathway by preventing the binding of Wnt with LRP5/6 $(25,26)$. In the Wnt/ $\beta$-catenin signaling pathway, GSK-3 $\beta$ is thought to phosphorylate without Wnt signaling, and consequently induces the degradation of $\beta$-catenin, thus, decreasing the expression of cyclin D1. Our results suggested that the expression of $\beta$-catenin and cyclin D1 was partly inhibited by DKK-1, which further demonstrates that ABPS promote chondrocyte proliferation through the $W n t / \beta$-catenin singaling pathway.

In conclusion, our results demonstrate that ABPS activate the $\mathrm{Wnt} / \beta$-catenin signaling pathway, thus contributing to chondrocyte proliferation. However, further studies are required using animals to verify this conclusion. In our study, we also found that ABPS increased the expression of type II collagen in the chondrocytes, which suggests that ABPS inhibits cartilage degradation by increasing the expression of type II collagen, a major secretory molecule of the ECM.

\section{Acknowledgements}

The present study was supported by the National Natural Science Foundation of China (grant no. 81373818\&81102609), the Special Research Fund for Doctor Discipline in College (20123519110001), the Key Natural Sciences Foundation of Fujian Province (2014Y0032), and the Natural Science Foundation of Fujian Province (2014J01357).

\section{References}

1. Sulzbacher I: Osteoarthritis: histology and pathogenesis. Wien Med Wochenschr 163: 212-219, 2013.
2. Chen C, Tambe DT, Deng L and Yang L: Biomechanical properties and mechanobiology of the articular chondrocyte. Am J Physiol Cell Physiol 305: C1202-C1208, 2013.

3. Clouet J, Vinatier C, Merceron C, et al: From osteoarthritis treatments to future regenerative therapies for cartilage. Drug Discov Today 14: 913-925, 2009.

4. Schroeppel JP, Crist JD, Anderson HC and Wang J: Molecular regulation of articular chondrocyte function and its significance in osteoarthritis. Histol Histopathol 26: 377-394, 2011.

5. Ciorba A and Martini A: Tissue engineering and cartilage regeneration for articular reconstruction. Int J Pediatr Otorhinolaryngol 70: 1507-1515, 2006.

6. Aman A, Nguyen M and Piotrowski T: Wnt/ $\beta$-catenin dependent cell proliferation underlies segmented lateral line morphogenesis. Dev Biol 349: 470-482, 2011.

7. Teo JL and Kahn M: The Wnt signaling pathway in cellular proliferation and differentiation: a tale of two coactivators. Adv Drug Deliv Rev 62: 1149-1155, 2010.

8. Chen FP, Chang CM, Hwang SJ, Chen YC and Chen FJ: Chinese herbal prescriptions for osteoarthritis in Taiwan: analysis of national health insurance dataset. BMC Complement Altern Med 14: 91, 2014.

9. Chen Q, Liu Z and He J: Achyranthes bidentata polysaccharide enhances immune response in weaned piglets. Immunopharmacol Immunotoxicol 31: 253-260, 2009.

10. Yu F, Li X, Cai L, et al: Achyranthes bidentata polysaccharides induce chondrocyte proliferation via the promotion of the G1/S cell cycle transition. Mol Med Rep 7: 935-940, 2013.

11. Li H, Li X, Liu G, et al: Bauhinia championi (Benth.) Benth. polysaccharides upregulate Wnt $/ \beta$-catenin signaling in chondrocytes. Int J Mol Med 32: 1329-1336, 2013.

12. Lim JC, Kania KD, Wijesuriya H, et al: Activation of $\beta$-catenin signalling by GSK-3 inhibition increases p-glycoprotein expression in brain endothelial cells. J Neurochem 106: 1855-1865, 2008.

13. Poole AR: Biochemical/immunochemical biomarkers of osteoarthritis: utility for prediction of incident or progressive osteoarthritis. Rheum Dis Clin North Am 29: 803-818, 2003.

14. Elsaid KA and Chichester CO: Review: collagen markers in early arthritic diseases. Clin Chim Acta 365: 68-77, 2006.

15. Yu Z, Che J, Ma X and He JM: Effect of Aloe vera polysaccharides on immunity and antioxidant activities in oral ulcer animal models. Carbohyd Polym 75: 307-311, 2009.

16. Chen Y, Shen Z and Chen X: Modulatory effect of Ganoderma lucidum polysaccharides on serum antioxidant enzymes activities in ovarian cancer rats. Carbohyd Polym 78: 258-262, 2009.

17. Bijlsma JW, Berenbaum F and Lafeber FP: Osteoarthritis an update with relevance for clinical practice. Lancet 377 : 2115-2126, 2011.

18. Gallelli L, Galasso $\mathrm{O}$ and Falcone $\mathrm{D}$, et al: The effects of nonsteroidal anti-inflammatory drugs on clinical outcomes, synovial fluid cytokine concentration and signal transduction pathways in knee osteoarthritis. A randomized open label trial. Osteoarthritis Cartilage 21: 1400-1408, 2013.

19. Flood J: The role of acetaminophen in the treatment of osteoarthritis. Am J Manag Care 16: S48-S54, 2010.

20. Bannuru RR, Vaysbrot EE, Sullivan MC and McAlindon TE: Relative efficacy of hyaluronic acid in comparison with NSAIDs for knee osteoarthritis: a systematic review and meta-analysis. Semin Arthritis Rheum 43: 593-599, 2013.

21. Kang M, Jung I, Hur J, et al: The analgesic and anti-inflammatory effect of WIN-34B, a new herbal formula for osteoarthritis composed of Lonicera japonica Thunb and Anemarrhena asphodeloides BUNGE in vivo. J Ethnopharmacol 131: 485-496, 2010.

22. Tao HY, He B, Liu SQ, et al: Effect of carboxymethylated chitosan on the biosynthesis of NGF and activation of the Wnt/ $\beta$-catenin signaling pathway in the proliferation of Schwann cells. Eur J Pharmacol 702: 85-92, 2013.

23. MacDonald BT, Tamai $\mathrm{K}$ and $\mathrm{He} \mathrm{X}$ : Wnt//-catenin signaling: components, mechanisms, and diseases. Dev Cell 17: 9-26, 2009.

24. Kim W, Kim M and Jho EH: Wnt/ $\beta$-catenin signalling: from plasma membrane to nucleus. Biochem J 450: 9-21, 2013.

25. Bafico A, Liu G, Yaniv A, Gazit A and Aaronson SA: Novel mechanism of Wnt signaling inhibition mediated by Dickkopf-1 interaction with LRP6/Arrow. Nat Cell Biol 3: 683-686, 2001.

26. Semenov MV, Tamai K, Brott BK, Kuhl M, Sokol S and He X: Head inducer Dickkopf-1 is a ligand for Wnt coreceptor LRP6. Curr Biol 11: 951-961, 2001. 